\title{
Symmetries and reversing symmetries of area-preserving polynomial mappings in generalised standard form
}

\author{
John A. G. Roberts ${ }^{1,2)}$ and Michael BaAkE ${ }^{3)}$
}

1): Department of Mathematics, La Trobe University, VIC 3086, Australia.

2): School of Mathematics, The University of New South Wales, Sydney NSW 2052, Australia;

email: jagr@maths.unsw.edu.au

3): Institut für Mathematik und Informatik, Universität Greifswald, Friedrich-Ludwig-Jahn-Str. 15a, 17487 Greifswald, Germany; email: mbaake@uni-greifswald.de

\begin{abstract}
We determine the symmetries and reversing symmetries within $\mathcal{G}$, the group of real planar polynomial automorphisms, of area-preserving nonlinear polynomial maps $L$ in generalised standard form,

$$
L: \quad x^{\prime}=x+p_{1}(y), \quad y^{\prime}=y+p_{2}\left(x^{\prime}\right),
$$

where $p_{1}$ and $p_{2}$ are polynomials. We do this by using the amalgamated free product structure of $\mathcal{G}$. Our results lead to normal forms for polynomial maps in generalised standard form and to a classification of the group structures of the reversing symmetry groups for such maps.
\end{abstract}




\section{Introduction}

In a series of recent articles [1, 3, 19], the symmetries and reversing symmetries of some dynamical systems (automorphisms) have been investigated systematically by means of algebraic methods. An automorphism $L$ of some space is said to have a symmetry if there exists an automorphism $S$ satisfying:

$$
S \circ L \circ S^{-1}=L
$$

and a reversing symmetry if there exists an automorphism $R$ satisfying

$$
R \circ L \circ R^{-1}=L^{-1} \text {. }
$$

The set of symmetries is non-empty (it certainly contains all powers of $L$ ) and this set is actually a group, the symmetry group $\mathcal{S}(L)$. On the other hand, the existence a priori of any reversing symmetries for a particular $L$ is unknown. When $L$ has a reversing symmetry, we call it reversible, and irreversible otherwise.

We denote by $\mathcal{R}(L)$ the set of all symmetries and reversing symmetries of $L$. It too is a group, called the reversing symmetry group [12 of $L$ (see also [9]). If $L$ has no reversing symmetries or if $L$ is an involution (i.e. $L^{2}=i d$ ), then $\mathcal{R}(L)=\mathcal{S}(L)$. On the other hand, if $R$ is a reversing symmetry then so is e.g. $S^{i} \circ R$ and $R \circ S^{i}, i \in \mathbb{Z}$ (in particular, $L^{i} \circ R=R \circ L^{-i}$ are reversing symmetries), whereas $R^{2 i}$ is a symmetry. More technically, if $L$ has reversing symmetries and is not an involution itself, $\mathcal{S}(L)$ is a normal subgroup of $\mathcal{R}(L)$ with $\mathcal{R}(L) / \mathcal{S}(L) \simeq C_{2}$. If, in addition, $L$ has an involutory reversing symmetry $R$ (i.e. $R^{2}=i d$ ) then $\mathcal{R}(L) \simeq \mathcal{S}(L) \times{ }_{s} C_{2}$ (it follows from (2) that the reversing symmetries $L^{i} \circ R$ and $R \circ L^{i}$ are then also involutions)円. In many cases of reversible automorphisms (and also in the analogous continuous-time case of reversible flows), it is in fact found that all reversing symmetries $R$ satisfying (2) are involutions. In this case, the automorphism $L$ can be written as the composition of two involutions, e.g. $L \circ R$ and $R$, or $R$ and $R \circ L$. The references [18 and [13] include reviews of the properties and applications of reversible automorphisms and flows.

In the papers [1, 19], the structure of $\mathcal{R}(L)$ has been identified when $L$, and possible symmetries $S$ and reversing symmetries $R$, belong to the group of linear automorphisms of the two-dimensional torus (or closely related groups). In this case, the solutions of (1) and (2) can be solved exactly using group theoretic and number theoretic techniques. In [3], extensions of some of these results are made for toral automorphisms in higher dimensions.

In this paper, we consider equations (11) and (2) for real polynomial maps $L$ of the plane in so-called generalised standard form:

$$
L: \quad x^{\prime}=x+p_{1}(y), \quad y^{\prime}=y+p_{2}\left(x^{\prime}\right),
$$

\footnotetext{
${ }^{1}$ We denote the cyclic group of order $n$ by $C_{n}$ and use $\times_{s}$ to denote a semi-direct product.
} 
where $p_{1}$ and $p_{2}$ are (real) polynomials (and the new $x$ coordinate $x^{\prime}$ needs to be calculated first so it can be substituted into the second equation). The form of (3) guarantees that the generalised standard form of map is always area-preserving (even with $p_{1}$ and $p_{2}$ replaced with non-polynomial functions). As such, this form has often been used to create and study models of area-preserving mappings [14, 15, 18]. Two other standard forms of area-preserving maps, known as the McMillan and de Vogelaere forms, can always be transformed to generalised standard form [18, Section 3.2]. For instance, the McMillan map

$$
M: \quad x^{\prime}=y, \quad y^{\prime}=-x+f(y),
$$

under the transformation $x \mapsto x, y \mapsto x-y$ becomes (3) with $p_{1}(y)=-y$ and $p_{2}\left(x^{\prime}\right)=2 x^{\prime}-f\left(x^{\prime}\right)$.

The much-studied area-preserving Hénon map in McMillan form is:

$$
H_{1}: \quad x^{\prime}=y, \quad y^{\prime}=-x+2 y(\lambda+y),
$$

and in generalised standard form is:

$$
H_{2}: \quad x^{\prime}=x-y, \quad y^{\prime}=y+2 x^{\prime}\left(1-\lambda-x^{\prime}\right),
$$

where $\lambda$ is a parameter. The Hénon map (6) is the simplest nonlinear polynomial example of (3) and all area-preserving quadratic polynomial mappings can be reduced to it [10]. A McMillan map (44) is always reversible with e.g. involutory reversing symmetries: $R: x^{\prime}=y, y^{\prime}=x$ and $M \circ R: x^{\prime}=x, y^{\prime}=-y+f(x)$ (so that $M$ is the composition of involutions $M \circ R$ and $R$ ). Hence $H_{1}$ is certainly reversible and, since reversibility is invariant under conjugation, $H_{2}$ is reversible with e.g. involutory polynomial reversing symmetries $R: x^{\prime}=x, y^{\prime}=-y+2 x(1-\lambda-x)$ or $R \circ H_{2}$ : $x^{\prime}=x-y, y^{\prime}=-y$ (so that $H_{2}$ is the composition of involutions $R$ and $R \circ H_{2}$ ). The reversibility of $H_{1}$ or $H_{2}$ has been much exploited in numerical studies because it makes it easier to find periodic orbits [14, 15, 18].

For $L$ of the form (3), the inverse map $L^{-1}$ is given by

$$
L^{-1}: \quad x^{\prime}=x-p_{1}\left(y^{\prime}\right), \quad y^{\prime}=y-p_{2}(x) .
$$

Hence, if $p_{1}$ and $p_{2}$ are polynomial, then $L$ is an example of a polynomial automorphism of $\mathbb{R}^{2}$ to itself since both $L$ and its inverse are polynomial. The polynomial automorphisms of $\mathbb{R}^{2}$ to itself form a group $\mathcal{G}$ (often called the general affine group $G A_{2}$ or the affine Cremona group [7]). In [8], the term generalised Hénon transformation is used for the map

$$
H_{g}: x^{\prime}=y, \quad y^{\prime}=-\delta x+p(y)
$$


with $p$ a polynomial function satisfying $\operatorname{deg} p \geq 2$ and $\delta \neq 0$ the constant Jacobian determinant of $H_{g}$. Moreover, it is shown that any nonlinear element of $\mathcal{G}$ which is dynamically non-trivial is conjugate within $\mathcal{G}$ to a composition

$$
H_{g_{n}} \circ H_{g_{n-1}} \circ \ldots H_{g_{2}} \circ H_{g_{1}}
$$

$n \geq 1$, of generalised Hénon transformations with $H_{g_{i}}$ depending on the constant $\delta_{i}$ and polynomial $p_{i}(y)$. Some of the dynamical properties of (9) have been studied in 8, 4, 6].

Although the symmetries and reversing symmetries of (9) will be studied more fully elsewhere, the generalised standard form (3) is a particular, and significant, areapreserving case of (9) when $n=2$, further justifying its study (see [2] and Remark 5 below in Section 5). Therefore, in this paper, we restrict possible symmetries $S$ or reversing symmetries $R$ of $L$ in (3) to also be polynomial automorphisms and, with this restriction, we are able to solve (11) and (2) exactly.

Our main results, Theorem 1 and its Corollary, are presented in the next Section. The key property that allows us to obtain our results in this paper is that the group $\mathcal{G}$ of polynomial automorphisms has a known structure. This structure is described in Section 3 and its usefulness in deciding conjugacy problems like (1) and (2) is pointed out. In Section 4, we give the details behind the proof of Theorem 1. Finally, in Section 5 , we discuss the implications of our results, the possible group structures of $\mathcal{R}(L)$ for $L$ a polynomial map of generalised standard form, and some extensions of the work presented here that will appear elsewhere [2].

\section{Statement of results}

We restrict ourselves to the dynamically interesting case of (3) when $L$ is nonlinear and consider separately the two cases when both $p_{1}$ and $p_{2}$ are nonlinear and when just one of $p_{1}$ or $p_{2}$ is nonlinear. Our main result is:

Theorem 1 Let $L$ be a nonlinear polynomial mapping of generalised standard form (3). Then $L$ possesses a non-trivial symmetry $S \neq L^{k}, k \in \mathbb{Z}$, or a reversing symmetry $R$ that is also a polynomial automorphism if and only if $p_{i}$ satisfy the conditions of Table 1 (if $\left.\operatorname{deg} p_{i} \geq 2, i=1,2\right)$ or Table 2 (if $\left.\operatorname{deg} p_{1}=1, \operatorname{deg} p_{2} \geq 2\right)$. In each case, the corresponding symmetries or reversing symmetries are as indicated. The case when $\operatorname{deg} p_{2}=1, \operatorname{deg} p_{1} \geq 2$ follows from Table 2 with replacements $x \rightarrow y$ and $p_{2} \rightarrow-p_{1}$.

We reiterate from the Introduction that because the symmetries and reversing symmetries form a group, other symmetries and reversing symmetries follow from combin-

ing the generating ones presented in Tables 1 and 2 (the groups generated by them are 


\begin{tabular}{|ll|l|}
\hline conditions on $p_{1}$ and $p_{2}$ of $L$ & symmetry $(S) /$ reversing symmetry $(R)$ \\
\hline \hline S1: & $\begin{array}{l}p_{1} \text { odd around } e / 2 \\
p_{2} \text { odd around } c / 2\end{array}$ & $S_{1}: x^{\prime}=-x+c, y^{\prime}=-y+e \quad\left(S_{1}^{2}=i d\right)$ \\
\hline S2: & $\begin{array}{l}p_{1} \text { arbitrary } \\
p_{2}(y)=\frac{1}{a} p_{1}\left(\frac{y}{a}+e\right)\end{array}$ & $S_{2}: x^{\prime}=a y-e a, y^{\prime}=\frac{x+p_{1}(y)}{a}+e \quad\left(S_{2}^{2}=L\right)$ \\
\hline S3: & $\begin{array}{l}p_{1} \text { odd around } \frac{1}{2}\left(e-\frac{c}{a}\right) \\
p_{2}(y)=-\frac{1}{a} p_{1}\left(\frac{y-c}{a}\right)\end{array}$ & $S_{3}: x^{\prime}=a y+c, y^{\prime}=-\frac{x+p_{1}(y)}{a}+e \quad\left(S_{3}^{2}=S_{1} \circ L\right)$ \\
\hline \hline R1: & $\begin{array}{l}p_{1} \text { arbitrary } \\
p_{2} \text { odd around } c / 2\end{array}$ & $\begin{array}{l}R_{1}: x^{\prime}=-x-p_{1}(y)+c, y^{\prime}=y \quad\left(R_{1}^{2}=i d\right) \\
L \circ R_{1}: x^{\prime}=-x+c, y^{\prime}=y-p_{2}(x)\end{array}$ \\
\hline R2: & $\begin{array}{l}p_{1} \text { odd around } e / 2 \\
p_{2} \text { arbitrary }\end{array}$ & $\begin{array}{l}R_{2}: x^{\prime}=x+p_{1}(y), y^{\prime}=-y+e \quad\left(R_{2}^{2}=i d\right) \\
L \circ R_{2}: x^{\prime}=x, y^{\prime}=-y+e+p_{2}(x)\end{array}$ \\
\hline R3: & $\begin{array}{l}p_{1} \text { even around } e / 2 \\
p_{2} \text { even around } c / 2\end{array}$ & $\begin{array}{l}R_{3}: x^{\prime}=-x-p_{1}(y)+c, y^{\prime}=-y+e \quad\left(R_{3}^{2}=i d\right) \\
L \circ R_{3}: x^{\prime}=-x+c, y^{\prime}=-y+e+p_{2}\left(x^{\prime}\right)\end{array}$ \\
\hline R4: & $\begin{array}{l}p_{1} \text { arbitrary } \\
p_{2}(y)=-\frac{1}{a} p_{1}\left(\frac{y}{a}+e\right)\end{array}$ & $\begin{array}{l}R_{4}: x^{\prime}=a y-e a, y^{\prime}=\frac{x}{a}+e \quad\left(R_{4}^{2}=i d\right) \\
L \circ R_{4}: x^{\prime}=a\left(y-e-p_{2}(x)\right), y^{\prime}=\frac{x}{a}+e+p_{2}\left(x^{\prime}\right)\end{array}$ \\
\hline R5: & $p_{1}$ odd around $\frac{1}{2}\left(e-\frac{c}{a}\right)$ \\
$p_{2}(y)=\frac{1}{a} p_{1}\left(\frac{y-c}{a}\right)$ & $R_{5}: x^{\prime}=a y+c, y^{\prime}=-\frac{x}{a}+e \quad\left(R_{5}^{4}=i d\right)$ \\
\hline
\end{tabular}

Table 1: Necessary and sufficient conditions for polynomial map $L$ of the form (33) with $\operatorname{deg} p_{i} \geq 2$ to have a non-trivial symmetry $S$ or reversing symmetry $R$. The parameters $a, c$ and $e$ are arbitrary. In the case of S3 where $S_{3}^{2}=S_{1} \circ L$, the $S_{1}$ here is $x^{\prime}=-x+(c+a e), y^{\prime}=-y+\left(e-\frac{c}{a}\right)$, since in this case $p_{1}$ is odd around $\frac{1}{2}\left(e-\frac{c}{a}\right)$ and $p_{2}$ is odd around $\frac{1}{2}(c+a e)$. 


\begin{tabular}{|ll|l|}
\hline \multicolumn{2}{|c|}{ condition on polynomial $p_{2}$ of $L$} & symmetry $(S) /$ reversing symmetry $(R)$ \\
\hline \hline S1: & $p_{2}$ odd around $e / 2$ & $S_{1}: x^{\prime}=-x+e, y^{\prime}=-y-2 B / A \quad\left(S_{1}^{2}=i d\right)$ \\
\hline \hline \multirow{2}{*}{ R $: \quad p_{2}$ odd around $e / 2$} & $R_{1}: x^{\prime}=-x+e, y^{\prime}=y-p_{2}(x) \quad\left(R_{1}^{2}=i d\right)$ \\
& & $R_{1} \circ L: x^{\prime}=-x-p_{1}(y)+e, y^{\prime}=y$ \\
\hline \multirow{2}{*}{ R2: } & $p_{2}$ arbitrary & $R_{2}: x^{\prime}=x, y^{\prime}=-y+p_{2}(x)-2 B / A \quad\left(R_{2}^{2}=i d\right)$ \\
& & $R_{2} \circ L: x^{\prime}=x+p_{1}(y) y^{\prime}=-y-2 B / A$ \\
\hline
\end{tabular}

Table 2: Necessary and sufficient conditions for polynomial map $L$ of the form (3) with $p_{1}(y)=A y+B, \operatorname{deg} p_{2} \geq 2$ to have a non-trivial symmetry $S$ or reversing symmetry $R$. The parameter $e$ is arbitrary.

detailed in Section 5, cf. Tables 5 and 6). In particular, in the cases of reversibility R1-R4 of Table 1 and R1-R2 of Table 2, the reversing symmetry $R_{i}$ in these Tables is easily checked to be an involution and we also list the involutory reversing symmetry $L \circ R_{i}$ or $R_{i} \circ L$ for the given maps $L$ (with $L$ being the composition of involutions $L \circ R_{i}$ with $R_{i}$ in Table 1 or $R_{i}$ with $R_{i} \circ L$ in Table 2). In the case R5 of reversibility in Table 1 , the reversing symmetry $R_{5}$ has order 4 . All possible reversing symmetries of $L$ are orientation-reversing, except in cases R3 and R5 of Table 1 where $R$ is orientation-preserving.

Note that a necessary condition for symmetry possibilities S2 and S3 and reversibility possibilities R4 and R5 of Table 1 is $\operatorname{deg} p_{1}=\operatorname{deg} p_{2}$. In the case of $\mathrm{S} 2$ and $\mathrm{S} 3$, the non-trivial symmetry $S_{2}\left(S_{3}\right)$ is a square root of $L\left(S_{1} \circ L\right)$. More detailed remarks on the results contained in Tables 1 and 2 will be made in Section 5 .

Under an affine transformation

$$
x \mapsto \alpha x+\beta, \quad y \mapsto \gamma y+\delta,
$$

the mapping $L$ of (3) transforms to $L^{\prime}$ which is also of generalised standard form:

$$
L^{\prime}: \quad x^{\prime}=x+\alpha p_{1}\left(\frac{y-\delta}{\gamma}\right), \quad y^{\prime}=y+\gamma p_{2}\left(\frac{x^{\prime}-\beta}{\alpha}\right) .
$$

Such transformations can be used to bring the cases of $L$ of Tables 1 and 2 into more transparent normal forms. Since possession of a symmetry or reversing symmetry is invariant under conjugation, the symmetries or reversing symmetries of Tables 1 and 2 transform under the same affine transformation used on $L$.

Corollary 1 A polynomial automorphism L of generalised standard form (3) possesses a symmetry $S$ or reversing symmetry $R$ that is also a polynomial automorphism if and 


\begin{tabular}{|c|c|c|}
\hline \multicolumn{2}{|c|}{ conditions on $p_{1}$ and $p_{2}$ of $L$} & symmetry $(S)$ / reversing symmetry $(R)$ \\
\hline S1: & $\begin{array}{l}p_{1} \text { odd around } 0 \\
p_{2} \text { odd around } 0\end{array}$ & $S_{1}: x^{\prime}=-x, \quad y^{\prime}=-y \quad\left(S_{1}^{2}=i d\right)$ \\
\hline $\mathrm{S} 2:$ & $\begin{array}{l}p_{1} \text { arbitrary } \\
p_{2}=p_{1}\end{array}$ & $S_{2}: x^{\prime}=y, \quad y^{\prime}=x+p_{1}(y) \quad\left(S_{2}^{2}=L\right)$ \\
\hline S3: & $\begin{array}{l}p_{1} \text { odd around } 0 \\
p_{2}=-p_{1}\end{array}$ & $S_{3}: x^{\prime}=y, \quad y^{\prime}=-x-p_{1}(y) \quad\left(S_{3}^{2}=-L\right)$ \\
\hline R1: & $\begin{array}{l}p_{1} \text { arbitrary } \\
p_{2} \text { odd around } 0\end{array}$ & $\begin{array}{l}R_{1}: x^{\prime}=-x-p_{1}(y), \quad y^{\prime}=y \quad\left(R_{1}^{2}=i d\right) \\
L \circ R_{1}: x^{\prime}=-x, \quad y^{\prime}=y-p_{2}(x)\end{array}$ \\
\hline $\mathrm{R} 2:$ & $\begin{array}{l}p_{1} \text { odd around } 0 \\
p_{2} \text { arbitrary }\end{array}$ & $\begin{array}{l}R_{2}: x^{\prime}=x+p_{1}(y), \quad y^{\prime}=-y \quad\left(R_{2}^{2}=i d\right) \\
L \circ R_{2}: x^{\prime}=x, \quad y^{\prime}=-y+p_{2}(x)\end{array}$ \\
\hline R3: & $\begin{array}{l}p_{1} \text { even around } 0 \\
p_{2} \text { even around } 0\end{array}$ & $\begin{array}{l}R_{3}: x^{\prime}=-x-p_{1}(y), \quad y^{\prime}=-y \quad\left(R_{3}^{2}=i d\right) \\
L \circ R_{3}: x^{\prime}=-x, \quad y^{\prime}=-y+p_{2}(x)\end{array}$ \\
\hline $\mathrm{R} 4:$ & $\begin{array}{l}p_{1} \text { arbitrary } \\
p_{2}=-p_{1}\end{array}$ & $\begin{array}{l}R_{4}: x^{\prime}=y, \quad y^{\prime}=x \quad\left(R_{4}^{2}=i d\right) \\
L \circ R_{4}: x^{\prime}=y+p_{1}(x), y^{\prime}=x-p_{1}\left(x^{\prime}\right)\end{array}$ \\
\hline R5: & $\begin{array}{l}p_{1} \text { odd around } 0 \\
p_{2}=p_{1}\end{array}$ & $R_{5}: x^{\prime}=y, \quad y^{\prime}=-x \quad\left(R_{5}^{4}=i d\right)$ \\
\hline
\end{tabular}

Table 3: Normal forms for polynomial map $L$ of the form (3) with $\operatorname{deg} p_{i} \geq 2$ when $L$ possesses a symmetry $S$ or reversing symmetry $R$ that is a polynomial automorphism.

only if, up to an affine transformation (10), $p_{i}$ satisfy the conditions of Table 3 (if $\left.\operatorname{deg} p_{i} \geq 2\right)$ or Table $4\left(\right.$ if $\left.\operatorname{deg} p_{1}=1, \operatorname{deg} p_{2} \geq 2\right)$. In each case, the corresponding symmetries or reversing symmetries are then as indicated.

Explicit affine transformations (10) that convert the cases of Table 11 into the corresponding ones of Table 3 are: S1 and R3 $\left(\alpha=\gamma=1, \beta=-\frac{c}{2}, \delta=-\frac{e}{2}\right) ; \mathrm{S} 2\left(\alpha=\frac{1}{a}\right.$, $\beta=e, \gamma=1, \delta=0)$; S3 and R5 $\left(\alpha=1, \beta=-\frac{1}{2}(c+a e), \gamma=a, \delta=-\frac{a}{2}\left(e-\frac{c}{a}\right)\right)$; R1 $\left(\alpha=\gamma=1, \beta=-\frac{c}{2}, \delta=0\right)$; R2 $\left(\alpha=\gamma=1, \beta=0, \delta=-\frac{e}{2}\right)$; R4 $\left(\alpha=\frac{1}{a}, \beta=e, \gamma=1\right.$, $\delta=0$ ). The conversion between Table 2 and Table 1 is achieved by (10) with: S1 and $\mathrm{R} 1\left(\alpha=1, \beta=-\frac{e}{2}, \gamma=A, \delta=B\right)$; $2(\alpha=1, \beta=0, \gamma=A, \delta=B)$. In all cases, the $p_{1}$ and $p_{2}$ of Table 3 and Table 4 equal the transformed functions shown in (11). 


\begin{tabular}{|ll|l|}
\hline \multicolumn{2}{|c|}{ condition on $p_{2}$ of $L$} & symmetry $(S) /$ reversing symmetry $(R)$ \\
\hline \hline S1: & $p_{2}$ odd around 0 & $S_{1}: x^{\prime}=-x, y^{\prime}=-y \quad\left(S_{1}^{2}=i d\right)$ \\
\hline \hline \multirow{2}{*}{ R1: } & $p_{2}$ odd around 0 & $R_{1}: x^{\prime}=-x, y^{\prime}=y-p_{2}(x) \quad\left(R_{1}^{2}=i d\right)$ \\
& & $R_{1} \circ L: x^{\prime}=-x-y, y^{\prime}=y$ \\
\hline \multirow{2}{*}{ R2: } & $p_{2}$ arbitrary & $R_{2}: x^{\prime}=x, y^{\prime}=-y+p_{2}(x) \quad\left(R_{2}^{2}=i d\right)$ \\
& & $R_{2} \circ L: x^{\prime}=x+y, y^{\prime}=-y$ \\
\hline
\end{tabular}

Table 4: Normal forms for polynomial map $L$ of the form (3) with $\operatorname{deg} p_{1}=1, \operatorname{deg} p_{2} \geq$ 2 when $L$ possesses a symmetry $S$ or reversing symmetry $R$ that is a polynomial automorphism. In each case of the Table, $L$ is given by $x^{\prime}=x+y, y^{\prime}=y+p_{2}\left(x^{\prime}\right)$.

The sufficiency of some of the conditions in Table 3 for allowing a symmetry or reversing symmetry of (3), irrespective of $p_{1}$ and $p_{2}$ being polynomials, has been noted previously in [14, Sections 1.2.4.7, 1.1.4.3] (cases S1, R1 and R2) and [17] (case R4). In fact, it is easy to check the sufficiency of all the (reversing) symmetry conditions of Tables 1- 目, irrespective of $p_{1}$ and $p_{2}$ being polynomials.

\section{$3 \quad$ Algebraic structure of $\mathcal{G}$}

Let $g$ be a polynomial mapping of $\mathbb{R}^{2}$ (or $\mathbb{C}^{2}$ ) of the form:

$$
g: \quad x^{\prime}=P(x, y), \quad y^{\prime}=Q(x, y)
$$

with $P(x, y)$ and $Q(x, y)$ polynomial in $x$ and $y$. Suppose $g$ is a bijection (i.e. one-toone and onto). Then $g$ has a well-defined inverse $g^{-1}$. Suppose $g^{-1}$ is also a polynomial mapping. Then $g$ is called a polynomial automorphism of the real (or complex) plane. The set of polynomial automorphisms of the plane forms a group $\mathcal{G}$ with a known structure, and this is the key ingredient that we exploit here. The mathematical context is the theory of amalgamated free products, convenient sources for background material are [5, Ch. 1.4] and [16, Sec. 4.2]

Specifically, let $\mathcal{A}$ denote the set of affine planar maps with $a \in \mathcal{A}$ of the form:

$$
a: \quad x^{\prime}=a_{11} x+a_{12} y+b_{1}, \quad y^{\prime}=a_{21} x+a_{22} y+b_{2},
$$

together with the invertibility condition of its linear part,

$$
a_{11} a_{22}-a_{12} a_{21} \neq 0 .
$$


It is not hard to see that $\mathcal{A}$ is a (six dimensional) subgroup of $\mathcal{G}$, as we have $\mathcal{A} \simeq$ $K^{2} \times{ }_{s} \mathrm{GL}(2, K)$ with $K=\mathbb{R}$ or $K=\mathbb{C}$.

Another (infinite-dimensional) subgroup $\mathcal{E}$ of $\mathcal{G}$ comprises the so-called elementary maps

$$
e: \quad x^{\prime}=\alpha x+p(y), y^{\prime}=\beta y+\gamma,
$$

with $\alpha \beta \neq 0$ and $p(y)$ a polynomial in $y$. Each $e \in \mathcal{E}$ has an inverse of similar form (explicitly, $e^{-1}$ is given by (15) with $\beta$ replaced by $\beta^{-1}$ and $\gamma$ by $-\beta^{-1} \gamma$ ).

Notice that the subgroups $\mathcal{A}$ and $\mathcal{E}$ intersect in those elementary maps that are also affine, which occurs whenever $e$ from (15) has affine $p$, i.e. $p(y)=c_{1} y+c_{0}$. We define the set $\mathcal{I}$ by

$$
\mathcal{I}=\mathcal{A} \cap \mathcal{E}
$$

and note that this intersection is again a subgroup of $\mathcal{G}$.

Now, we need the following result of Jung [11], see also [8, p. 68].

Theorem 2 [Jung] The group $\mathcal{G}$ of polynomial automorphisms of $\mathbb{R}^{2}$ (or $\mathbb{C}^{2}$ ) is the amalgamated free product of its subgroups $\mathcal{A}$ and $\mathcal{E}$, understood as groups with real (complex) parameters. More explicitly, $\mathcal{G}$ is the free product of $\mathcal{A}$ and $\mathcal{E}$ amalgamated along their intersection $\mathcal{I}$, i.e. $\mathcal{G}=\mathcal{A}_{\mathcal{I}}^{*} \mathcal{E}$.

For the present purpose, the practical meaning of Theorem 2 is that any $g \in \mathcal{G} \backslash \mathcal{I}$ can be written as a so-called reduced word

$$
g=g_{n} \circ g_{n-1} \circ \ldots \circ g_{1}
$$

where $n \geq 1$ and the $g_{i}$ alternate in belonging to $\mathcal{A} \backslash \mathcal{I}$ or $\mathcal{E} \backslash \mathcal{I}$. The value of $n \geq 1$ is called the length of the reduced word. Note that no reduced word is equal to the identity of $\mathcal{G}$, see $[8$, Cor. 2.2]. Observe that $g \in \mathcal{A} \backslash \mathcal{I}$ implies that also $s \circ g$ and $g \circ s$ are in $\mathcal{A} \backslash \mathcal{I}$, for arbitrary $s \in \mathcal{I}$, and analogously for $g \in \mathcal{E} \backslash \mathcal{I}$. The algebraic property of amalgamated free products then gives us the following structure for reduced words, see [8, Cor. 2.3] or [5, Thms. 25 and 26].

Proposition 1 The word expression (17) for $g \in \mathcal{G} \backslash \mathcal{I}$ is unique up to the modification that we can replace $g_{i}$, for any $i>1$, by $g_{i} \circ s$, with any $s \in \mathcal{I}$, and simultaneously replace $g_{i-1}$ by $s^{-1} \circ g_{i-1}$. Consequently, if $g \in \mathcal{G} \backslash \mathcal{I}$ can be represented both by $g_{n} \circ g_{n-1} \circ \ldots \circ g_{1}$ and $g_{n^{\prime}}^{\prime} \circ g_{n^{\prime}-1}^{\prime} \circ \ldots \circ g_{1}^{\prime}$, then $n=n^{\prime}$ and $g_{i}$ and $g_{i}^{\prime}$ both belong to either $\mathcal{A} \backslash \mathcal{I}$ or $\mathcal{E} \backslash \mathcal{I}$.

As an example, consider the generalised standard form (3). Let the mapping $t$ be given by

$$
t: \quad x^{\prime}=y, \quad y^{\prime}=x
$$


and, for $i=1,2$, define the following maps:

$$
\begin{aligned}
e_{i}: & x^{\prime}=x+p_{i}(y), \quad y^{\prime}=y, \\
q_{i}: & x^{\prime}=y, \quad y^{\prime}=x+p_{i}(y), \\
r_{i}: & x^{\prime}=x, \quad y^{\prime}=y+p_{i}(x) .
\end{aligned}
$$

Notice that $q_{i}=t e_{i}$ (we suppress the symbol $\circ$ from now on). We see the following: $t \in \mathcal{A} \backslash \mathcal{I} ; e_{i} \in \mathcal{E} \backslash \mathcal{I}$ if $\operatorname{deg} p_{i} \geq 2$ and $e_{i} \in \mathcal{I}$ if $\operatorname{deg} p_{i}=1$ or $0 ; q_{i} \in \mathcal{A} \backslash \mathcal{I}$ only if $\operatorname{deg} p_{i}=1$ or 0 ; and $r_{i} \in \mathcal{A} \backslash \mathcal{I}$ only if $\operatorname{deg} p_{i}=1$ and $r_{i} \in \mathcal{I}$ if $\operatorname{deg} p_{i}=0$.

Possible forms of reduced words (17) for (3) depend on the nature of $\operatorname{deg} p_{i}$ and we classify them as follows:

Type I: If $\operatorname{deg} p_{i} \geq 2$, then

$$
L=t e_{2} t e_{1}
$$

Type II: If $\operatorname{deg} p_{2} \geq 2$ and $\operatorname{deg} p_{1} \leq 1$, then

$$
L=t e_{2} q_{1}
$$

Type III: If $\operatorname{deg} p_{1} \geq 2$ and $\operatorname{deg} p_{2}=1$, then

$$
L=r_{2} e_{1}
$$

Type IV: If $\operatorname{deg} p_{1} \geq 2$ and $\operatorname{deg} p_{2}=0$, then

$$
L: x^{\prime}=x+p_{1}(y) y^{\prime}=y+K,
$$

with $K$ a constant, is itself an element of $\mathcal{E} \backslash \mathcal{I}$.

From (11) and (2), existence of a symmetry $S$ (reversing symmetry $R$ ) implies a conjugacy between $L$ and itself (its inverse $L^{-1}$ ). If we look for possible (reversing) symmetries that are themselves polynomial with polynomial inverse, then the conjugacy question is asked within $\mathcal{G}$. In this respect, the known group structure of $\mathcal{G}$ also helps. We say that the reduced word (17) is cyclically reduced if $n=1$ or if $g_{n}$ and $g_{1}$ do not both belong to $\mathcal{A} \backslash \mathcal{I}$ or $\mathcal{E} \backslash \mathcal{I}$.

Let us recall [16, Thm. 4.6(iii)], compare also [5, Thm. 26] and [5, Prop. 27].

Proposition 2 Let $g$ and $h$ be cyclically reduced elements of $\mathcal{G}$ that are conjugate in $\mathcal{G}$. If $h=h_{n} h_{n-1} \ldots h_{1}$ with $n \geq 2$ and $h_{i}, h_{i+1}$ as well as $h_{n}$ and $h_{1}$ belong to different factors, then $g=s\left\{h_{n} h_{n-1} \ldots h_{1}\right\} s^{-1}$ where $s \in \mathcal{I}$ and $\left\{h_{n} h_{n-1} \ldots h_{1}\right\}$ means some cyclic permutation of the $n$ letters $h_{i}$.

Finally, we also need the following result (with $\mathbb{R}^{*}=\mathbb{R} \backslash\{0\}$ ). 
Lemma 1 Let $p(x)$ be a non-constant real polynomial satisfying the identity:

$$
p(x)=\gamma p(\alpha x+\beta),
$$

for some $\gamma, \alpha \in \mathbb{R}^{*}$ and $\beta \in \mathbb{R}$. Then we have:

(i) if $\alpha=1$, then $\gamma=1$ and $\beta=0$, while no restrictions apply to $p(x)$ (trivial case);

(ii) if $\alpha$ is not a root of unity (i.e. $\alpha \neq \pm 1$ ), then $\gamma \alpha^{n}=1$ for some $n \geq 1$ and $p(x)=c\left(x+\frac{\beta}{\alpha-1}\right)^{n}$, with arbitrary $\beta \in \mathbb{R}$ and $c \in \mathbb{R}^{*}$.

The remaining case is $\alpha=-1$ which implies $\gamma^{2}=1$. In particular:

(iii) if $\alpha=-1$ and $\gamma=1$, then $\beta$ is arbitrary and $p(x)$ is even around $\beta / 2$, i.e. $p(x)=p(\beta / 2)+\sum_{n=1}^{N} c_{2 n}(x-\beta / 2)^{2 n}, c_{2 n} \in \mathbb{R}, \operatorname{deg} p=2 N$;

(iv) if $\alpha=\gamma=-1$, then $\beta$ is arbitrary and $p(x)$ is odd around $\beta / 2$, i.e. $p(x)=\sum_{n=0}^{N} c_{2 n+1}(x-\beta / 2)^{2 n+1}, c_{2 n+1} \in \mathbb{R}, \operatorname{deg} p=2 N+1$.

Proof: Let $\xi$ be a (real or complex) zero of $p(x)$. Let $A(x)$ be the affine map $A: x \mapsto \alpha x+\beta$. The identity (24) implies that $A^{n}(\xi)$ is also a zero of $p(x)$, for all integer $n \geq 1$. Since $p(x)$ has only finitely many zeros, the orbit $\left\{A^{n}(\xi) \mid n \in \mathbb{Z}^{+}\right\}$ must be eventually periodic. So we are led to study the finite orbits under iteration of $\mathcal{A}$.

Let $\alpha=1$ in $A(x)$. Then $A^{n}(\xi)=\xi+n \beta$. All orbits of $A^{n}$ are infinite and aperiodic unless $\beta=0$, in which case $A(x)$ is the identity. Substituting $\alpha=1$ and $\beta=0$ in (24) gives $\gamma=1$ since $p(x)$ is not the zero polynomial. This gives the trivial case (i).

Suppose $\alpha \neq 1$. Then the dynamics of $A(x)$ is conjugate to that of the linear mapping $L: z \mapsto \alpha z$ via the coordinate transformation $z=x+\frac{\beta}{\alpha-1}$. Since $L^{n}(z)=\alpha^{n} z$, we see that, for $\alpha$ real, there are two types of behaviour. The first occurs if $\alpha \neq-1$, in which case $L$ has only one periodic orbit, the fixed point $z=0$, whence $A$ has only one periodic orbit, the fixed point $\xi=-\frac{\beta}{\alpha-1}$. It follows that if $\alpha \neq \pm 1, p(x)$ has only one repeated zero over $\mathbb{C}$, the real zero $\xi=-\frac{\beta}{\alpha-1}$, so necessarily $p(x)$ takes the form $c\left(x+\frac{\beta}{\alpha-1}\right)^{n}$, where $n \geq 1$ (since $p$ is non-constant by assumption) and $c \in \mathbb{R}^{*}$ is arbitrary. Substituting this form into (24) reveals that we must have $\gamma \alpha^{n}=1$ and that no restrictions apply to $\beta$. This gives case (ii).

If $\alpha=-1$, we obtain the second possibility. Then $A(x)=-x+\beta$ is an involution, so every point has period 2 except for the fixed point at $\beta / 2$. Eq. (24) can now be written as $p\left(\frac{\beta}{2}+x\right)=\gamma p\left(\frac{\beta}{2}-x\right)$. Evaluating this at $x=0$ we see that either $\gamma=1$ or $p(\beta / 2)=0$. If $\gamma=1, p$ must be even around $\beta / 2$, but no further restrictions apply. This gives case (iii). Finally, let $\gamma \neq 1$, so $\xi=\beta / 2$ is a root of $p$. If we write $p$ as a polynomial in powers of $(x-\beta / 2)$ we see, by comparison of coefficients, that only $\gamma=-1$ remains, and that $p$ must then be odd around $\beta / 2$. This gives case (iv). 
REMARK: Lemma 1 has a natural complex counterpart. Cases (i) and (ii) are the same, except that one now has to exclude all roots of unity in (ii). Next, if $\alpha \neq 1$ is a root of unity, i.e. $\alpha^{n}=1$ for some $n \geq 2$, then $\gamma^{m}=1$ for some $m \mid n$ because iteration of (24) leads to the equation

$$
p\left(\frac{\beta}{1-\alpha}+x\right)=\gamma^{k} p\left(\frac{\beta}{1-\alpha}+\alpha^{k} x\right)
$$

for all $k \geq 1$. Consequently, cases (iii) and (iv) are to be replaced by appropriate cyclic symmetry requirements around $\frac{\beta}{1-\alpha}$.

\section{Proof of main result}

To prove our Theorem 11 of Section 2, we consider the above-mentioned Type I (20) and Type II (21) word forms for $L$ in polynomial generalised standard form and use the conjugacy result Proposition 2. In this Section, we will write our general element of $\mathcal{I}$ as

$$
s: \quad x^{\prime}=a x+b y+c, \quad y^{\prime}=d y+e,
$$

with $a d \neq 0$.

\section{Type I words}

Let us first consider the reversibility of Type I words (20), when both $p_{1}$ and $p_{2}$ are nonlinear. We have

$$
L=t e_{2} t e_{1} \Rightarrow L^{-1}=e_{1}^{-1} t e_{2}^{-1} t
$$

noting that $t$ is an involution so that $t^{-1}=t$. The word for $L$, and hence that for $L^{-1}$, is cyclically reduced. Assume that $L$ is reversible, so that $L$ and $L^{-1}$ are conjugate in $\mathcal{G}$. Using Proposition 2, we must have:

$$
L=t e_{2} t e_{1}=s t e_{2}^{-1} t e_{1}^{-1} s^{-1}
$$

or

$$
L=t e_{2} t e_{1}=s t e_{1}^{-1} t e_{2}^{-1} s^{-1} .
$$

Notice that $t e_{2}^{-1} t e_{1}^{-1}$ and $t e_{1}^{-1} t e_{2}^{-1}$ are the two cyclic permutations of $L^{-1}$ that begin with an element from $\mathcal{A} \backslash \mathcal{I}$ (by Proposition 1 we must reject the cyclic permutations that begin with an element from $\mathcal{E} \backslash \mathcal{I}$ because this is preserved on adding an $s$ from the left and disagrees with the word for $L$ which starts with an element of $\mathcal{A} \backslash \mathcal{I}$ ). 
If (27) holds, a reversing symmetry is:

$$
R=s e_{1}
$$

since (27) can be rewritten

$$
L=t e_{2} t e_{1}=s t e_{2}^{-1} t e_{1}^{-1} s^{-1}=s e_{1}\left(e_{1}^{-1} t e_{2}^{-1} t\right) e_{1}^{-1} s^{-1}=R L^{-1} R^{-1},
$$

and (2) with $R$ replaced by $R^{-1}$ is an equivalent definition of a reversing symmetry. Similarly, if (28) holds, a reversing symmetry is:

$$
R=s t
$$

since (28) can be rewritten

$$
L=t e_{2} t e_{1}=s t e_{1}^{-1} t e_{2}^{-1} s^{-1}=s t\left(e_{1}^{-1} t e_{2}^{-1} t\right) t s^{-1}=R L^{-1} R^{-1} .
$$

In the case (29), $R$ is nonlinear; in the case (31), $R$ is affine.

The leading element from $\mathcal{A} \backslash \mathcal{I}$ on the left hand side of (27) is $t$, whereas the leading element from $A \backslash \mathcal{I}$ on the right hand side is st (the same holds for (28)). Since the left and right hand sides are different reduced words for the same element $L$, it follows from Proposition 1 that:

$$
t=s t s^{\prime}
$$

where $s^{\prime} \in \mathcal{I}$. Writing out (33) readily shows that the coefficient of $y$ in the $x^{\prime}$ part of $s$ and $s^{\prime}$ vanishes. Consequently, we can take $b=0$ in (25) for $s$ in both (27) and (28).

Consider now the identity (27) in more detail. Using (18), (19) and (25) with $b=0$, the $x$ component of the right hand side of (27) equals

$$
x-a p_{1}\left(\frac{y-e}{d}\right)
$$

and the $y$ component is

$$
y-d p_{2}\left(\frac{x-c}{a}-p_{1}\left(\frac{y-e}{d}\right)\right)
$$

Comparing (34) to the $x$ component of $L$ in (3) shows that $p_{1}(y)$ satisfies:

$$
p_{1}(y)=-a p_{1}\left(\frac{y-e}{d}\right)
$$

Comparing (35) to the $y$ component of $L$ in (3) and using (36) shows that $p_{2}(y)$ satisfies:

$$
p_{2}\left(x+p_{1}(y)\right)=-d p_{2}\left(\frac{x+p_{1}(y)-c}{a}\right),
$$


or, equivalently,

$$
p_{2}(x)=-d p_{2}\left(\frac{x-c}{a}\right)
$$

The analysis of the second type of reversibility for a Type I map, described by (28), follows close to that above. Writing out both sides of (28), again with $b=0$ in $s$, and equating now leads to the conditions:

$$
p_{1}(y)=-a p_{2}\left(\frac{y-e}{d}\right)
$$

and

$$
p_{2}(y)=-d p_{1}\left(\frac{y-c}{a}\right)
$$

These show that a necessary condition for this type of reversibility is that $\operatorname{deg} p_{1}=$ $\operatorname{deg} p_{2}$. This can also be seen algebraically from (28) and Proposition 1 since corresponding elements belonging to $\mathcal{E} \backslash \mathcal{I}$ on the left and right hand sides of (28) must have the same degree (and $\operatorname{deg} e_{i}^{-1}=\operatorname{deg} e_{i}$ ).

It remains to study the conditions (36) and (37) and (38) and (39). Conditions (36) and (37) are both examples of the identity (24), and Lemma 1 can be used to work through the possible values for $a, c, d$ and $e$ in $s$ of (25), together with the conditions on $p_{1}$ and $p_{2}$. For example, taking case (i) of Lemma 1 for $p_{1}(y)$ in (36) imposes $a=-1, d=1$ and $e=0$, which implies in (37) that $p_{2}(x)=-p_{2}(-x+c)$. Hence $p_{2}$ is odd around $c / 2$ for arbitrary $c, s: x^{\prime}=-x+c, y^{\prime}=y$ from (25), and from (29) we obtain the reversing symmetry $R_{1}$ shown in R1 of Table 1. In a similar way, taking case (iii) or (iv) of Lemma 1 for $p_{1}(y)$ gives, respectively, cases R3 and R2 of Table 1 . Taking case (ii) of Lemma 11 for $p_{1}(y)$ in (36) does not produce a further example of reversibility. It implies taking $d \neq \pm 1, a d^{-n}=-1$ and $e$ arbitrary. Substituting into (37) shows that for $p_{2}$, only case (ii) of Lemma 1 is possible, and only if $a \neq \pm 1$ and $d a^{-n^{\prime}}=-1$. The two conditions $a d^{-n}=-1$ and $d a^{-n^{\prime}}=-1$ imply $(-1)^{n^{\prime}} d^{n n^{\prime}-1}=-1$ which cannot occur since $d \neq \pm 1$ and $n \geq 2, n^{\prime} \geq 2$ by the assumption $\operatorname{deg} p_{i} \geq 2$.

The conditions (38) and (39) imply

$$
p_{1}(y)=a d p_{1}\left(\frac{y-(e+d c)}{a d}\right)
$$

another case of the identity (24) in which it is readily seen case (i) and case (iv) of Lemma 1 are the only possibilities. Taking case (i) for $p_{1}$ in (40) implies $a d=1$, $e+d c=0$ and $p_{1}$ arbitrary. Substituting in (39) gives $p_{2}$ defined as in R4 of Table 1 . The reversing symmetry $R_{4}$ in this instance follows from (31) with $s: x^{\prime}=a x-e a, y^{\prime}=$ $y / a+e$. Similarly, taking case (iv) of Lemma 1 for (40) gives R5 of Table 1 . 
Finally, consider the symmetries of $L$ when it is a Type I word (20). We now need to investigate the necessary and sufficient conditions for $L=t e_{2} t e_{1}$ to be conjugate to itself in $\mathcal{G}$. Using Proposition 2, we must either have:

$$
L=t e_{2} t e_{1}=s t e_{2} t e_{1} s^{-1}
$$

in which case a symmetry is obviously $S=s$, or

$$
L=t e_{2} t e_{1}=s t e_{1} t e_{2} s^{-1}
$$

in which case a symmetry is

$$
S=s t e_{1}
$$

The analysis in each case is identical to the corresponding conditions for reversibility modulo a sign change (stemming from the fact that $e_{i}^{-1}$ is obtained from $e_{i}$ by $p_{i} \rightarrow$ $-p_{i}$ ). For (41) to hold, the conditions are

$$
p_{1}(y)=a p_{1}\left(\frac{y-e}{d}\right), \quad p_{2}(y)=d p_{2}\left(\frac{y-c}{a}\right) .
$$

For (42) to hold, the conditions are

$$
p_{1}(y)=a p_{2}\left(\frac{y-e}{d}\right), \quad p_{2}(y)=d p_{1}\left(\frac{y-c}{a}\right) .
$$

The analysis of (44) and (45) is identical to the corresponding conditions for reversibility and uses Lemma 1. Conditions (44) yield S1 of Table 1 (obtained from taking case (iv) of Lemma 1 for $p_{1}$ ) and conditions (45) yield S2 and S3 of Table 1 .

\section{Type II words}

We consider the (reversing) symmetries of a Type II word (21) in the case that $\operatorname{deg} p_{2} \geq$ 2 and $\operatorname{deg} p_{1}=1$. Such a word is not cyclically reduced since $t$ and $q_{1}$ both belong to $A \backslash \mathcal{I}$. Consequently, we work with such a word after conjugation with $t$ (and also call this transformed word $L$ for convenience) :

$$
L=t\left(t e_{2} q_{1}\right) t=e_{2} r_{1}: \quad x^{\prime}=x+p_{2}\left(y^{\prime}\right), \quad y^{\prime}=y+p_{1}(x)
$$

which is now cyclically reduced. Conjugation by $t$ involves interchange of $x$ and $y$ in the original map. Using Proposition 2 and Proposition [1, we must have:

$$
L=e_{2} r_{1}=s e_{2}^{ \pm 1} r_{1}^{ \pm 1} s^{-1}
$$


with the + sign taken when considering symmetries and the - sign for reversibility. Clearly, a symmetry is given by the affine transformation $s$ whereas a reversing symmetry is given by

$$
R=s e_{2}^{-1}
$$

Writing out (47) with $s$ given by (25) and taking $p_{1}=A x+B, A \neq 0$, for the affine function $p_{1}$, shows that $b=0$ in $s$ and:

$$
p_{1}(a x+c)= \pm d p_{1}(x)
$$

together with

$$
p_{2}(d y+e)= \pm a p_{2}(y) .
$$

Substituting $p_{1}(x)=A x+B$ into (49) gives information about the parameters of the mapping $s$ :

$$
a= \pm d, \quad c=(a-1) B / A
$$

Consequently, (50) becomes

$$
p_{2}(y)=\frac{1}{d} p_{2}(d y+e)
$$

which is an example of (24) with $\alpha=d=1 / \gamma$ and $\beta=e$. Only case (i) $(d=1$, $e=0$ and $p_{2}$ arbitrary) and case (iv) $\left(d=-1, e\right.$ arbitrary and $p_{2}$ odd around $\left.e / 2\right)$ of Lemma 1 apply. Case (i) gives R2 of Table 2, using (51) and (48) to write out $R_{2}$, and similarly, case (iv) gives S1 and R1 of Table 2 (remembering to interchange $x$ and $y$ in order to get the results for $t e_{2} q_{1}$ ). This solves the problem of finding symmetries and reversing symmetries for Type II words with $\operatorname{deg} p_{1}=1$.

Note that the remaining case of nonlinear maps (3) after Type I and II words is when $p_{2}$ is affine and $p_{1}$ is nonlinear. This is a Type III word (22) $L=r_{2} e_{1}$ whose inverse is $L^{-1}=e_{1}^{-1} r_{2}^{-1}$. The (reversing) symmetries of the latter follow from those of $e_{2} r_{1}$, just considered, by the replacements $p_{2} \rightarrow-p_{1}, p_{1} \rightarrow-p_{2}$ and are precisely the (reversing) symmetries of $L=r_{2} e_{1}$ (because $L$ and $L^{-1}$, for arbitrary $L$, always have the same symmetries, and have the same reversing symmetries when these are involutions). This completes the proof of Theorem 1 .

\section{Remarks on results}

In this paper, we have calculated explicitly the symmetries and reversing symmetries within $\mathcal{G}$, the group of planar polynomial automorphisms, of a real nonlinear polynomial 
map $L$ in generalised standard form (3). The conditions for, and nature of, possible symmetries $S$ and reversing symmetries $R$ are given in Tables 1 and 2 . These results lead to normal forms for polynomial generalised standard maps with the corresponding (reversing) symmetries in Tables 3 and 1 . Some observations about the results in Tables 1-4 were already made following Theorem 1 and Corollary 1 1. We conclude with the following additional remarks:

Remark 1: Suppose $L$ is an element of $\mathcal{G}$ with a $k$ th root $M$ also belonging to $\mathcal{G}$, so $M^{k}=L$. Then, obviously, $M$ is a symmetry of $L$ in $\mathcal{G}$. It follows from our results that when $L$ is nonlinear and of generalised standard form, it can only have a square root in $\mathcal{G}$ and only when $p_{1}$ and $p_{2}$ are both nonlinear (because the only non-trivial symmetry $S_{1}$ of Tables 2 or 4 is a linear involution and $\left.\left(L \circ S_{1}\right)^{2}=L^{2}\right)$. Case S2 of Table 3 makes clear the only case of a square root that can occur at the level of the normal form (the conditions at Case S2 of Table 1 are the general necessary and sufficient conditions for this to occur). When the conditions in S3 of Table 1 or Table 3 are satisfied, $\mathrm{S} 1$ is also satisfied and it is seen that $S_{3}$ is a square root of $S_{1} \circ L$ and a fourth root of $L^{2}$ (since $S_{3}$ commutes with $S_{1}$ ) but neither of the polynomial automorphisms $S_{1} \circ L$ or $L^{2}$ are in generalised standard form.

Remark 2: Whenever $L$ is nonlinear and one of $p_{1}$ or $p_{2}$ is affine, it follows from R2 of Table 2 that $L$ is reversible in $\mathcal{G}$ since it always has an involutory reversing symmetry (e.g. the Hénon map in the form $H_{2}$ of (60). When both $p_{1}$ and $p_{2}$ are nonlinear, we need to examine the conditions of Table 1 to see whether $L$ is reversible but it is obvious that irreversibility is the generic case. In the special case $\operatorname{deg} p_{1}=$ $\operatorname{deg} p_{2}=2, L$ is always reversible since a quadratic polynomial is always even about some point and case R3 is satisfied (i.e. $p(x)=a x^{2}+b x+c=a\left(x+\frac{b}{2 a}\right)^{2}+\left(c-\frac{b^{2}}{4 a}\right)$ so $\left.p\left(x-\frac{b}{2 a}\right)=p\left(-x-\frac{b}{2 a}\right)\right)$. It turns out then that the lowest degree combination of $p_{1}$ and $p_{2}$ for which the conditions $\mathrm{R} i, i=1, \ldots, 5$ of Table 1 can be avoided is $\operatorname{deg} p_{1}=2$, $\operatorname{deg} p_{2}=3$ or vice versa. After [8], define the degree of a polynomial map (12) by $\operatorname{deg} g=\max \left(\operatorname{deg} x^{\prime}, \operatorname{deg} y^{\prime}\right)=\max (\operatorname{deg} P(x, y), \operatorname{deg} Q(x, y))$. It is seen that for a map of the form (3) $\operatorname{deg} L=\operatorname{deg} p_{1} \cdot \operatorname{deg} p_{2}$. Hence the least degree in which irreversibility in $\mathcal{G}$ occurs for area-preserving maps of generalized standard form is $\operatorname{deg} L=6$ (in [17], using the idea of local reversibility, an example is given of a map (3) of degree 9 that has no $C^{3}$ involutory reversing symmetry). More generally, for area-preserving maps $g$ in $\mathcal{G}$ that are not in the form (3), degree four is the minimum degree at which irreversibility occurs (see Remark 5 below).

Remark 3: Tables 3 and 1 help to make transparent the various possible structures of the reversing symmetry group $\mathcal{R}(L) \subset \mathcal{G}$. 
Firstly, consider the case that $p_{1}$ in $L$ is affine. From R2 of Table 6 , the reversible $L$ can be taken to be in the form $x^{\prime}=x+y, y^{\prime}=y+p_{2}\left(x^{\prime}\right)$. If $p_{2}$ in the latter is not odd around some value $e / 2$, then $\mathcal{S}(L)=\langle L\rangle \simeq C_{\infty}$ since S1 of Table 2 is not satisfied and $L$ has no root in $\mathcal{G}$ by Remark 1 above. Because $R_{2}: x^{\prime}=x, y^{\prime}=-y+p_{2}(x)$ of Table 国 is an involution, $\mathcal{R}(L)$ is a semidirect product of $\mathcal{S}(L)$ and the $C_{2}$ group generated by $R_{2}$ [1] (recall the dihedral group $D_{\infty} \simeq C_{\infty} \times_{s} C_{2}$ ). When $p_{2}$ is odd around some value $e / 2$, the symmetry group increases to accommodate the involutory symmetry $S_{1}$ of Table 2. Note that $R_{1}$ of Table 2 now becomes an additional reversing symmetry of $L$ and is the composition of $S_{1}$ with $R_{2}$. In fact, in this case, $R_{2}$ and $S_{1}$ commute and we can simplify the structure of $\mathcal{R}(L)$ to that of a direct product of its $D_{\infty}$ subgroup $\left(\langle L\rangle \times_{s}\left\langle R_{2}\right\rangle\right)$ and its $C_{2}$ subgroup $\left\langle S_{1}\right\rangle$. The situation is summarized in Table 0 .

Secondly, consider the case that $p_{1}$ and $p_{2}$ are both nonlinear. Table 6 summarizes the possible distinct symmetry group and reversing symmetry group structures and their generators. The top case of Table 6 corresponds to the absence of reversing symmetries and nontrivial symmetries. Whenever S2 or S3 of Table 1 hold in Table 6, the $C_{\infty}$ part of $\mathcal{S}(L)$ is generated by the corresponding $S_{2}$ or $S_{3}$ of Table 1 - otherwise the $C_{\infty}$ part is generated by $L$ itself. Table 6 illustrates various ways that the cases of Table 1 or Table 3 interrelate. For example, it is seen from Table 1 that whenever the conditions for S1 hold, then R1 and R2 follow automatically and the reversing symmetry $R_{2}$ is seen to be the composition of the $R_{1}$ together with the symmetry $S_{1}$. Also, whenever the conditions for R5 hold in Table 1, then S1, S2, R1 and R2 immediately follow. Furthermore, since a non-zero polynomial cannot be even about some point and odd about some (other) point, it follows that reversibility possibility R3 of Table 1 or Table 3 can only co-exist with R4 or S2, with any two of R3, R4 and S2 implying the third.

Recall that a necessary condition for symmetry possibilities S2 and S3 and reversibility possibilities $\mathrm{R} 4$ and $\mathrm{R} 5$ of Table $1 \mathrm{1}$ is $\operatorname{deg} p_{1}=\operatorname{deg} p_{2}$. It is also clear that symmetry possibilities S2 and S3 cannot co-exist. As found in the S1 possibility of Table 5, it is possible to simplify the structure of $\mathcal{R}(L)$ in Table 6 to a direct product for possibility S1;not S2 and possibility S3. This follows because $R_{1}$ and $S_{1}$ commute in these cases and, significantly in the case S3, because $R_{1}$ is a reversing symmetry of $S_{3}$ (as well as being a reversing symmetry of $L=S_{1} S_{3}^{2}$ ). Note in possibility S3, the reversing symmetry $R_{4}=S_{3} \circ R_{1}$. Interestingly, in possibility S1 and S2 of Table 6 we cannot achieve $\mathcal{R}(L)$ as a direct product because $R_{1}$ is a reversing symmetry of $L=S_{2}^{2}$ but not of $S_{2}$ (in fact, $R_{1} \circ S_{2} \circ R_{1}=S_{1} \circ S_{2}^{-1}$ ). In other words, $\left\langle S_{2}\right\rangle$ is not a normal subgroup of $\left\langle S_{2}, R_{1}\right\rangle$. The map $S_{2}$ is said to have $R_{1}$ as a reversing 2 -symmetry because $R_{1}$ conjugates $S_{2}^{2}$ to its inverse but not $S_{2}$ to its inverse. The reversing symmetry $R_{5}$ in possibility $\mathrm{S} 1$ and $\mathrm{S} 2$ is obtained from $R_{5}=S_{2} \circ R_{1}$. 


\begin{tabular}{|c|c|c|}
\hline $\mathcal{S}(L)$ & $\mathcal{R}(L)$ & Possibilities \\
\hline \hline$C_{\infty}$ & $C_{\infty} \times{ }_{s} C_{2} \simeq D_{\infty}$ & \\
$\langle L\rangle$ & $\langle L\rangle \times_{s}\left\langle R_{2}\right\rangle$ & not S1 \\
\hline$C_{\infty} \times C_{2}$ & $\left(C_{\infty} \times C_{2}\right) \times_{s} C_{2} \simeq D_{\infty} \times C_{2}$ & \\
$\langle L\rangle \times\left\langle S_{1}\right\rangle$ & $\left(\langle L\rangle \times{ }_{s}\left\langle R_{2}\right\rangle\right) \times\left\langle S_{1}\right\rangle$ & $\mathrm{S} 1$ \\
\hline
\end{tabular}

Table 5: Possible group structures of $\mathcal{S}(L)$ and $\mathcal{R}(L)$ with their generators when $L$ of (3) has one of $p_{1}$ or $p_{2}$ affine. The term 'S1' or 'not S1' refers to satisfaction or non-satisfaction of the condition listed for $\mathrm{S} 1$ in Table 2.

\begin{tabular}{|c|c|c|}
\hline $\mathcal{S}(L)$ & $\mathcal{R}(L)$ & Possibilities \\
\hline$C_{\infty}$ & $\mathcal{S}(L)$ & \\
\hline$\langle L\rangle$ & $\langle L\rangle$ & $\operatorname{not} \mathrm{S} i, i=1,2,3 ; \operatorname{not} \mathrm{R} i, i=1, \ldots, 5$ \\
\hline$\left\langle S_{2}\right\rangle$ & $\left\langle S_{2}\right\rangle$ & $\mathrm{S} 2 ; \operatorname{not} \mathrm{R} i, i=1, \ldots, 5$ \\
\hline$C_{\infty}$ & $C_{\infty} \times{ }_{s} C_{2} \simeq D_{\infty}$ & \\
\hline$\langle L\rangle$ & $\langle L\rangle \times{ }_{s}\left\langle R_{i}\right\rangle$ & not $\mathrm{S} i, i=1,2,3 ;$ one $\mathrm{R} i, i=1, \ldots, 4$ \\
\hline$\left\langle S_{2}\right\rangle$ & $\left\langle S_{2}\right\rangle \times_{s}\left\langle R_{3}\right\rangle$ & $\mathrm{S} 2$ and $\mathrm{R} 3(\Leftrightarrow \mathrm{S} 2$ and $\mathrm{R} 4)$ \\
\hline & $\begin{array}{l}\left(C_{\infty} \times C_{2}\right) \times{ }_{s} C_{2} \\
\quad \simeq D_{\infty} \times C_{2}\end{array}$ & \\
\hline$\langle L\rangle \times\left\langle S_{1}\right\rangle$ & $\left(\langle L\rangle \times_{s}\left\langle R_{1}\right\rangle\right) \times\left\langle S_{1}\right\rangle$ & $\mathrm{S} 1 ;$ not $\mathrm{S} 2(\Rightarrow \mathrm{R} 1$ and R2 $)$ \\
\hline$\left\langle S_{3}\right\rangle \times\left\langle S_{1}\right\rangle$ & $\left(\left\langle S_{3}\right\rangle \times_{s}\left\langle R_{1}\right\rangle\right) \times\left\langle S_{1}\right\rangle$ & $\mathrm{S} 3(\Rightarrow \mathrm{S} 1, \mathrm{R} 1, \mathrm{R} 2$ and $\mathrm{R} 4)$ \\
\hline $\begin{array}{c}C_{\infty} \times C_{2} \\
\left\langle S_{2}\right\rangle \times\left\langle S_{1}\right\rangle\end{array}$ & $\begin{array}{c}\left(C_{\infty} \times C_{2}\right) \times{ }_{s} C_{2} \\
\left(\left\langle S_{2}\right\rangle \times\left\langle S_{1}\right\rangle\right) \times{ }_{s}\left\langle R_{1}\right\rangle\end{array}$ & $\mathrm{S} 1$ and $\mathrm{S} 2(\Leftrightarrow \mathrm{R} 5 ; \Rightarrow \mathrm{R} 1$ and $\mathrm{R} 2)$ \\
\hline
\end{tabular}

Table 6: Possible group structures of $\mathcal{S}(L)$ and $\mathcal{R}(L)$ with their generators when $L$ of (3i) has both $p_{1}$ and $p_{2}$ nonlinear. The term 'S1' or 'not S1' etc. refers to the satisfaction or non-satisfaction of the condition listed for S1 in Table [1. 
Remark 4: Veselov [20, 21] has shown that an area-preserving map $L$ in $\mathcal{G}$ possesses a nonconstant polynomial or rational integral $I(x, y)=I\left(x^{\prime}, y^{\prime}\right)$ if and only if $L$ has a symmetry $S$ with $\langle L, S\rangle \simeq C_{\infty} \times C_{\infty}$. ¿From Tables 5 and 6 , this symmetry group structure does not occur for the polynomial generalised standard maps considered here so integrable cases are absent.

Remark 5: In future work [2], we will consider $\mathcal{R}(L)$ for arbitrary $L \in \mathcal{G}$, i.e. $L$ not necessarily in generalised standard form. There we can show that some of the results found here hold more generally. Furthermore, we can obtain these results using just algebraic properties of the amalgamated free product structure (rather than by means of constructive calculations for the standard form of $L$, as we did here). For instance, if $L \in \mathcal{G}$ is not conjugate to an affine map or to an elementary map, it can be shown that $\mathcal{S}(L) \subset \mathcal{G}$ is always an Abelian group of the form $C_{\infty}$ or $C_{\infty} \times C_{2}$. Furthermore, any reversing symmetries $R \in \mathcal{G}$ are involutions or of order 4 .

A necessary condition for $L \in \mathcal{G}$ to be reversible is that $L$ is area-preserving or anti area-preserving (i.e. $\operatorname{det} d L=-1$ ). To study reversibility, one can use from [8] the normal form (9) for a nonlinear (non-elementary) $L \in \mathcal{G}$. The degree of the normal form (9) can be shown [8] to be $\prod_{i} \operatorname{deg} p_{i}$, with $\operatorname{deg} p_{i} \geq 2$. When (9) has $n=1$ and $\delta_{1}=1$, the area-preserving mapping $H_{g_{1}}$ is precisely of McMillan form (田) and so is reversible. In particular, this implies whenever $\operatorname{deg} L$ is prime and $L$ is area-preserving, then $L$ is reversible in $\mathcal{G}$. If we consider the area-preserving case of (9) with $n=2$ (i.e. $\delta_{1} \delta_{2}=1$ ), we find that $H_{g_{2}} \circ H_{g_{1}}$ is reversible when $\operatorname{deg} p_{1} \neq \operatorname{deg} p_{2}$ if and only if: (i) $\delta_{1}=\delta_{2}=1$ (in which case $H_{g_{i}}$ are both (reversible) McMillan maps of the form (何) and $H_{g_{2}} \circ H_{g_{1}}$ always has e.g. the involutory reversing symmetry $\left.R: x^{\prime}=-x+p_{1}(y), y^{\prime}=y\right)$; or (ii) $\delta_{1}=\delta_{2}=-1$ (i.e. $H_{g_{2}} \circ H_{g_{1}}$ is in the generalised standard form (3)) and at least one of the conditions R1, R2 or R3 of Table 1 is satisfied. In particular, with respect to (ii), if $\operatorname{deg} p_{1}$ is odd and $\operatorname{deg} p_{2}$ is even or vice versa, Table 1 shows that there are no non-trivial symmetries and the only possibility for reversibility is one of R1 and R2.

When $\operatorname{deg} p_{1}=\operatorname{deg} p_{2}$, an area-preserving $H_{g_{2}} \circ H_{g_{1}}$ has, in addition to (i) and (ii), other possibilities to be reversible but these are not generically satisfied. This is true even for the least possible case of $\operatorname{deg}\left(H_{g_{2}} \circ H_{g_{1}}\right)=4$ (i.e. $\operatorname{deg} p_{1}=\operatorname{deg} p_{2}=2$ ) when the additional reversibility condition for $p_{1}(y)=y^{2}+C_{1}, p_{2}(y)=y^{2}+C_{2}$ in the $\left(\delta_{1}, C_{1}, C_{2}\right.$ )-space is: (iii) $C_{1}=\delta_{1}^{2 / 3} C_{2}$ (this form for $p_{1}$ and $p_{2}$ in $H_{g_{2}} \circ H_{g_{1}}$ can be taken without loss of generality from [8, Theorem 2.6]). Thus, considering quartic polynomial maps in $\mathcal{G}$ that are area-preserving, these are always reversible if they are derived from (9) with $n=1$ or are derived from (9) with $n=2$ and satisfy $\delta_{1}=\delta_{2}= \pm 1$ (noting, from Remark 2 above, that (3) is always reversible when $\operatorname{deg} L=4$ ). Otherwise, if they are derived from (9) with $n=2, \delta_{1} \delta_{2}=1$ and $\delta_{1} \neq \pm 1$, they are generically irreversible in $\mathcal{G}$. 


\section{Acknowledgements}

This work was supported by a La Trobe University Central Starter Grant and by the German Research Council (DFG).

\section{References}

[1] M. Baake and J.A.G. Roberts, Reversing symmetry group of $G L(2, \mathbb{Z})$ and $P G L(2, \mathbb{Z})$ matrices with connections to cat maps and trace maps, J. Phys. A 30 (1997) 1549-1573.

[2] M. Baake and J.A.G. Roberts, Reversing symmetry group of polynomial automorphisms of $\mathbb{R}^{2}$, in preparation.

[3] M. Baake and J.A.G. Roberts, Symmetries and reversing symmetries of toral automorphisms, Nonlinearity 14 (2001) R1-R24.

[4] E. Bedford and J. Smillie, Polynomial diffeomorphisms of $\mathbb{C}^{2}$. V. Critical points and Lyapunov exponents, J. Geom. Anal. 8 (1998) 349-383.

[5] D. E. Cohen, Combinatorial Group Theory: A Topological Approach, (Cambridge University Press, Cambridge, 1989).

[6] H. R. Dullin and J. D. Meiss, Generalized Hénon maps: the cubic diffeomorphisms of the plane, Physica D 143 (2000) 262-289.

[7] A. van den Essen, Seven lectures on polynomial automorphisms, in: Automorphisms of Affine Spaces, ed. A. van den Essen (Kluwer, Dordrecht, 1995) pp. 3-39.

[8] S. Friedland and J. Milnor, Dynamical properties of plane polynomial automorphisms, Ergod. Th. Ef Dyn. Syst. 9 (1989) 67-99.

[9] G. R. Goodson, Inverse conjugacies and reversing symmetry groups, Amer. Math. Monthly 106 (1999) 19-26.

[10] M. Hénon, Numerical study of quadratic area preserving mappings, Quart. Appl. Math. 27 (1969) 291-312.

[11] H.W.E. Jung, Über ganze birationale Transformationen der Ebene, J. Reine Angew. Math. 184 (1942) 161-174. 
[12] J. S. W. Lamb, Reversing symmetries in dynamical systems, J. Phys. A 25 (1992) 925-937.

[13] J. S. W. Lamb and J.A. G. Roberts, Time-reversal symmetry in dynamical systems: A survey, Physica D 112 (1998) 1-39.

[14] R. S. MacKay, Renormalisation in Area-Preserving Maps (World Scientific, Singapore, 1993), and references therein.

[15] R.S. MacKay and J.D. Meiss (eds.), Hamiltonian Dynamical Systems (Adam Hilger, Bristol, 1987).

[16] W. Magnus, A. Karrass, and D. Solitar, Combinatorial Group Theory, 2nd ed., (Dover, New York, 1976).

[17] J.A. G. Roberts and H.W. Capel, Area preserving mappings that are not reversible, Phys. Lett. A 162 (1992) 243-248.

[18] J. A. G. Roberts and G. R. W. Quispel, Chaos and time-reversal symmetry - order and chaos in reversible dynamical systems, Phys. Rep. 216 (1992) 63-177.

[19] J. A. G. Roberts and R. S. Wilson, Reversibility of orientation-reversing cat maps and the amalgamated free product structure of $\operatorname{PGL}(2, \mathbb{Z})$, preprint (2002).

[20] A. P. Veselov, Integrable maps, Russian Math. Surveys 46 (1991) 1-51.

[21] A. P. Veselov, Growth and integrability in the dynamics of mappings, Commun. Math. Phys. 145 (1992) 181-193. 